\title{
From wheelchair to walking: First case report of post-electrical burn destruction of patellar ligament with its one-stage reconstruction and restoration of function
}

\author{
Sandip Kanti Basu, Jayanta Bain, Bijay Kumar Majumdar, Debarati Chattopadhyay ${ }^{1}$, \\ Debasis Baitalik2, Yatindra Kumar Dewangan³, Debtanu Bhattacharya ${ }^{4}$, Pritha Rakshit, \\ Vivek Gupta, Ashwani Kumar, Rathindra Nath Haldar \\ Department of Plastic Surgery, Institute of Post Graduate Medical Education and Research, Departments of ${ }^{2}$ Plastic Surgery \\ and ${ }^{4}$ Burn Unit M.R. Bangur Hospital, Kolkata, West Bengal, ${ }^{1}$ Department Plastic Surgery, All India Institute of Medical \\ Sciences, Rishikesh, Uttarakhand, ${ }^{3}$ Department of Plastic Surgery, Ramkrishna Care Hospital, Raipur, Chhattisgarh, India
}

Address for correspondence: Dr. Jayanta Bain, Department of Plastic Surgery, Institute of Post Graduate Medical Education and Research, Kolkata - 741 163, West Bengal, India. E-mail: jbain212@gmail.com

\section{ABSTRACT}

Electrical burn injury causing bilateral patellar ligament destruction leading to complete loss of knee extension is a very rare injury. In such situation, surgical repair or reconstruction of the patellar ligament becomes necessary to restore knee functions. Here, we present such a case of an 8-year-old boy, whose both patellar ligaments were destroyed throughout its length due to high-voltage electrical injury. His left knee joint cavity was exposed and grossly infected, but the right knee joint cavity was apparently intact. The right-sided patellar ligament was reconstructed with an ipsilateral and looped semitendinosus tendon graft and covered with a medial gastrocnemius musculocutaneous flap. The patient had an uneventful recovery, and full range of motion in the right knee joint along with good bipedal locomotion was achieved successfully.

\section{KEY WORDS}

Electrical burn; patellar ligament; semitendinosus tendon graft

\section{INTRODUCTION}

njury to patellar ligament is one of the most common forms of tendon injury around knee joint. The resultant extension disability from patellar ligament rupture poses a significant surgical challenge to the surgeon. Rupture of patellar ligament may be acute, which

\begin{tabular}{|l|l|}
\hline \multicolumn{2}{|c|}{ Video Available on: www.ijps.org } \\
\hline \multicolumn{2}{|c|}{ Access this article online } \\
\hline Quick Response Code: & Website: \\
\hline
\end{tabular}

is commonly seen amongst young athletes, whereas chronic rupture is seen in cases of systemic diseases such as rheumatoid arthritis, systemic lupus erythematosus, chronic renal failure, hyperparathyroidism and diabetes mellitus..$^{[1,2]}$ Patellar ligament injury due to electrical burns is very rare, and we did not find a single case

This is an open access article distributed under the terms of the Creative Commons Attribution-NonCommercial-ShareAlike 3.0 License, which allows others to remix, tweak, and build upon the work non-commercially, as long as the author is credited and the new creations are licensed under the identical terms.

For reprints contact: reprints@medknow.com

How to cite this article: Basu SK, Bain J, Majumdar BK, Chattopadhyay D, Baitalik D, Dewangan YK, et al. From wheelchair to walking: First case report of post-electrical burn destruction of patellar ligament with its one-stage reconstruction and restoration of function. Indian J Plast Surg 2017;50:100-3. 
of severe and bilateral injury after extensive literature search. Here, we describe such a case and its unique mode of one-stage reconstruction, the first of its kind described in literature.

\section{CASE REPORT}

An 8-year-old boy was admitted in the burn unit of our hospital with the history of electrical burn injury to both of his knees when his lower limbs were entangled in a live electric wire. On examination, there was third-degree burn on both knees anteriorly with near full-thickness destruction of patellar ligament on both sides. The patient was initially managed conservatively. After multiple debridements over next 4 weeks, resultant defects on left side consisted of complete loss of patellar ligament, loss of some part of the upper end of tibia due to osteomyelitis and exposure of the joint space. On the right side also, patellar ligament was destroyed, tubercle of tibia and lower pole of patella were exposed, but the joint cavity seemed to be intact [Figure 1].

At this point of time, as both patellar ligaments were destroyed, the young boy was unable to extend his knees actively, thus he became wheelchair bound for locomotion. Hence, to give the boy an opportunity to walk, reconstruction of patellar ligament, at least on one side, became essential. For the right knee, reconstruction of patellar tendon was done with an ipsilateral and looped semitendinosus tendon graft, and for the grossly destroyed left knee joint, arthrodesis was done.

Both the joints were operated in the same sitting by two teams simultaneously. The patient was operated

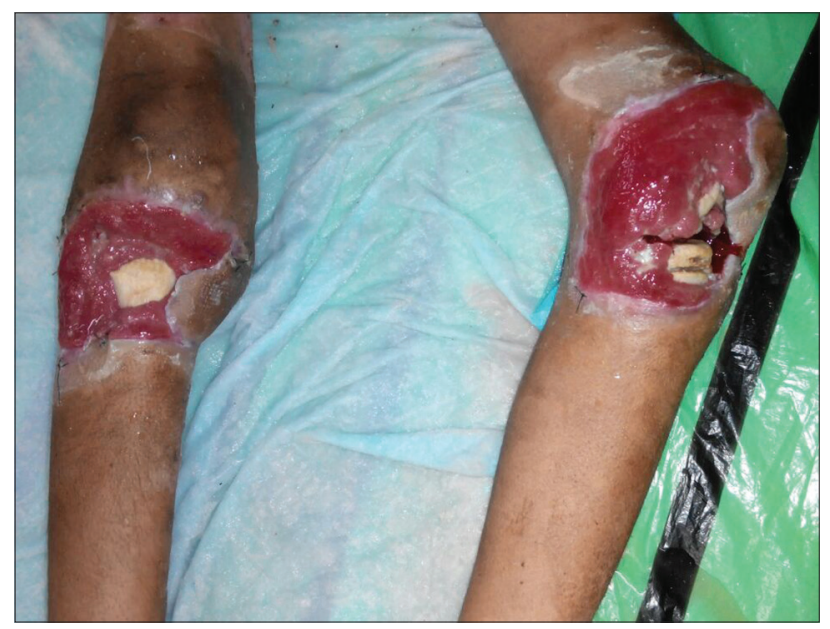

Figure 1: Clinical presentation before surgery in supine position under general anaesthesia. On the left-sided knee joint, infected and necrotic cartilages and bone of the articular surfaces of tibia and femur were removed; the bone ends were made raw and the exposed bones were covered with ipsilateral pedicled medial gastrocnemius muscle flap with skin graft over it.

On the right side, patellar tendon was reconstructed. Incision was made at pes anserinus. Semitendinosus tendon was identified and divided at musculotendinous junction. Proximal end of the divided distal part was delivered distally at incision site keeping the insertion intact. Mid pole of the patella and the tibial tuberosity were drilled separately to make a tunnel with a cannulated burr over a K-wire. Great care was taken so as not to damage the articular surface of the patella. Thereafter, the semitendinosus tendon was passed through patella from medial to lateral side and then passed in reverse direction through the tibial tunnel and fixed to distal intact insertion of semitendinosus, thus forming a loop; the tension of the reconstructed tendon was adjusted by sutured in $30^{\circ}$ of flexion of knee joint [Figure $2 \mathrm{a}$ and $\mathrm{b}$ ]. The whole reconstruction was covered with medial gastrocnemius musculocutaneous flap.

On the left side, physiotherapy for hip and ankle joint was started on the $4^{\text {th }}$ post-operative day. On the right side, knee joint was subjected to physiotherapy from $2^{\text {nd }}$ week onwards; he was given passive motion training, gradually increasing the range of motion and later active movements from second post-operative week to subsequent 12 weeks. For both sides, gradual

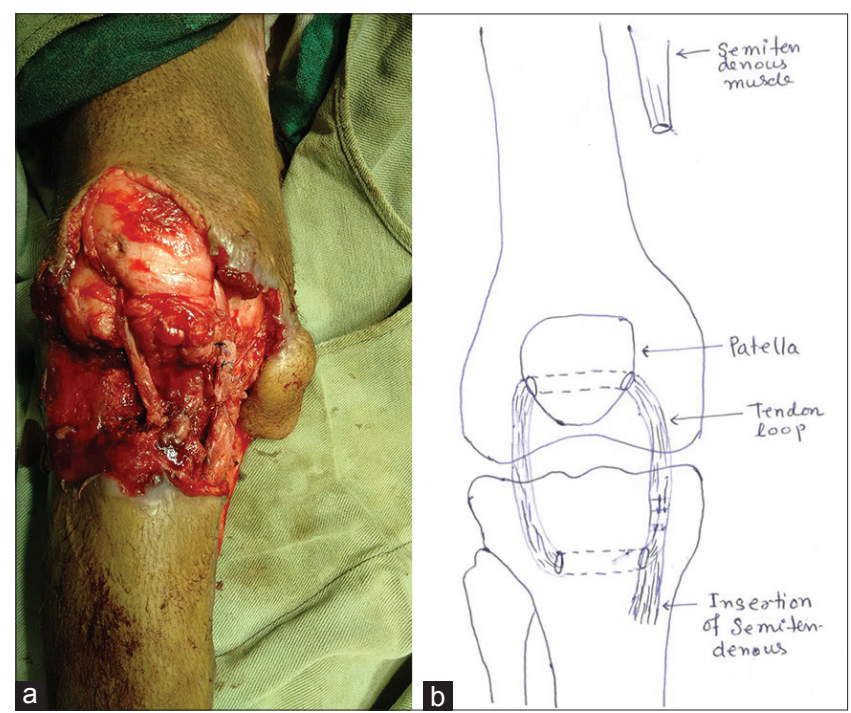

Figure 2: $(a$ and $b)$ Reconstruction patellar tendon by semitendinosus tendon 
weightbearing such as standing with help was started after 6 weeks postoperatively and walking with assistance started on $8^{\text {th }}$ post-operative week.

On follow-up after 10 months, the functional outcome was evaluated using Insall modification of Knee scoring (1993) system on the right side where the patellar tendon reconstruction was done. We found no flexion contracture, no pain, no extension lag, normal alignment (no varus or valgus deformity) of knee and total range of motion was $120^{\circ}$; with no anteroposterior or mediolateral instability. Thus, total Knee Society score was 72 out of 100 . The patient thus achieved a good range of motion of the right knee joint [Figure 3a and b] and walking without any assistance [Video 1].

\section{DISCUSSION}

Movement of knee joint is an integral part of human locomotion, and the patellar ligament has a major contribution to it. Patellar ligament is a strong tendon to hold the patella with the tibia so as to transmit the extensor force which provides the mechanism and strength of the extensor mechanism of knee. For normal bipedal locomotion, at least one knee joint has to be functional, without which it is not possible to walk unsupported.

In most instances of acute patellar ligament rupture, where the quality of tendon is good, primary repair can be done. However, for chronic cases, where primary repair is not possible, various different reconstruction techniques have been described such as autologous semitendinosus alone ${ }^{[3]}$ or with the gracilis graft, ${ }^{[4]}$ allograft using the tendoachiles ${ }^{[5]}$ or a contralateral bone-patellar-tendon-bone graft $^{[6]}$ and synthetic materials..$^{[2]}$ Although in our case, the injury was an acute

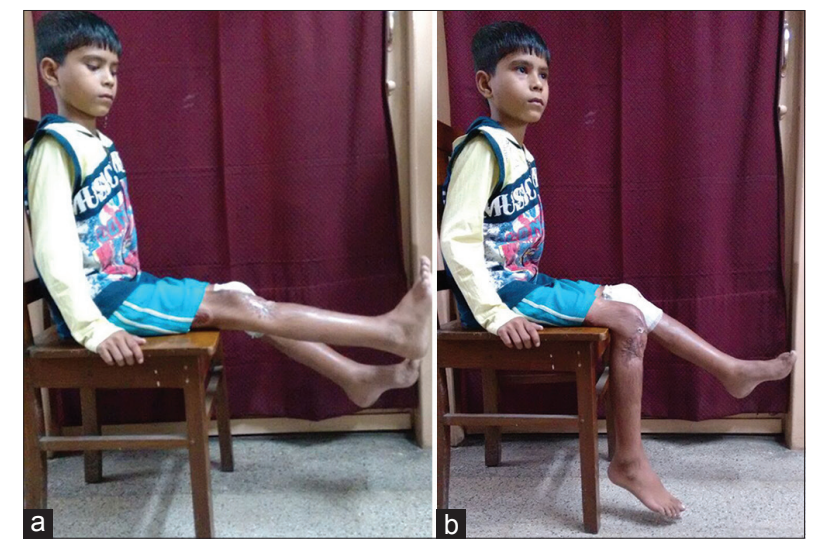

Figure 3: (a) Full extension of the left knee. (b) $90^{\circ}$ flexion of the left knee one, primary repair was impossible because there was a complete loss of patellar ligament which had to be reconstructed, preferably with autologous tissue in view of the infected condition. As there was wide area of soft tissue loss around the both knees, coverage of the knee was also a great concern. As the left knee joint cavity was partially destroyed, extensor apparatus reconstruction was not attempted in view of the possibility that pain-free full range of movement, the joint might not be achieved. Furthermore, the upper end of tibia was osteomyelitic, precluding the option of primary joint replacement.

As the right knee joint cavity was intact but with a completely destructed patellar ligament, we attempted to reconstruct the right-sided patellar tendon so that there is at least one well-functioning knee joint to give the boy a chance to walk. We used an ipsilateral semitendinosus tendon graft because it is a robust tissue which can restore the strength and stability of the extensor mechanism of the knee joint and it has minimum donor site morbidity. ${ }^{[7]}$ Furthermore, being an autograft, the ipsilateral semitendinosus tendon graft can be used safely in infected condition such as in this case. There are essentially two steps, harvesting and fixation. After dividing through the musculotendinous junction, some authors also prefer to cut the distal insertion and harvest it as a free tendon graft, which is then passed through the osseous tunnel made through the patella and the tibial tuberosity, and sometimes, the tibial passage is additionally strengthened using a bioabsorbable interference screw. ${ }^{[8]}$ However, we followed the alternative method ${ }^{[9]}$ where the distal insertion of the semitendinosus tendon was kept intact, and the proximal cut end was tunnelled through the patella and tibial tuberosity making a loop and sutured with itself. In view of existing infection, we used no metallic screw/wire to fix the tendon with the patella or tibia. We believe that the intact insertion of semitendinosus tendon with tibia is sufficient to hold it and preservation of distal insertion can facilitate the healing process. ${ }^{[4]}$

Our case is unique in its presentation and management. No such case of bilateral destruction of patellar tendon with extensive soft tissue injury around knee joints due to electrical burn has been described in the literature. Neither has been described previously, the reconstruction of patellar ligament in acute injury along with soft tissue coverage. Here, the semitendinosus loop was used to re-establish the extensor mechanism of one knee, along with gastrocnemius flap coverage to provide a one-stage 
reconstruction of the knee joint to give the boy a chance to walk.

\section{CONCLUSION}

To conclude, bilateral patellar tendon injury by electrical burn is very rare. In such cases, at least one patellar tendon needs to be reconstructed to achieve bipedal locomotion. An autologous looped semitendinosus graft for reconstruction of patellar ligament is a noble method, in such situations, which can restore the strength and stability and function of knee joint. This procedure has minimal morbidity, achieves full range of motions and develops enough strength for weightbearing so that patient can walk without assistance.

\section{Financial support and sponsorship}

Nil.

\section{Conflicts of interest}

There are no conflicts of interest.

\section{REFERENCES}

1. Amlang $\mathrm{MH}, \mathrm{Zwipp} \mathrm{H}$. Damage to large tendons: Achilles, patellar and quadriceps tendons. Chirurg 2006;77:637-49.

2. Naguib AM, Sefton GK. Recurrent bilateral rupture of the patellar tendons: Tendon replacement using polyester connective tissue prosthesis. Injury Extra 2006;37:379-82.

3. Jablonski JJ, Jarmuziewicz P, Druzbicki M. Reconstruction of chronic patellar tendon rupture with semitendinosus tendon: Case report. Ortop Traumatol Rehabil 2011;13:607-15.

4. Moretti L, Vicenti G, Abate A, Pesce V, Moretti B. Patellar tendon rerupture in a footballer: Our personal surgical technique and review of the literature. Injury 2014;45:452-6.

5. McNally PD, Marcelli EA. Achilles allograft reconstruction of a chronic patellar tendon rupture. Arthroscopy 1998;14:340-4.

6. Milankov MZ, Miljkovic N, Stankovic M. Reconstruction of chronic patellar tendon rupture with contralateral BTB autograft: A case report. Knee Surg Sports Traumatol Arthrosc 2007;15:1445-8.

7. Van der Bracht $H$, Verdonk R, Stuyts B. Augmentation of a patellar tendon repair with an autologous semitendinosus graft. Acta Orthop Belg 2009;75:417-9.

8. Van der Zwaal P, van Arkel E. Recurrent patellar tendon rupture: Reconstruction using ipsilateral gracilis and semitendinosus tendon autografts. Injury Extra 2007;38:320-3.

9. Nguene-Nyemb AG, Huten D, Ropars M. Chronic patellar tendon rupture reconstruction with a semitendinosus autograft. Orthop Traumatol Surg Res 2011;97:447-50. 